\title{
Desastres naturales y desigualdad: Evidencia en América Latina y El Caribe
}

URL: http://revistas.uta.edu.ec/erevista/index.php/bcoyu/article/view/684 DOI: http://dx.doi.org/10.31164/bcoyu.20.2019.684

\section{Desfrancois - Pierre ${ }^{1}$}

Fecha de recepción: 22 de febrero de 2019

\section{Resumen}

El presente trabajo de investigación explora la relación entre los desastres naturales y la desigualdad de ingresos en América Latina y El Caribe a partir de un modelo de datos de panel que cubre 14 países durante el periodo 2000-2017. El estudio econométrico revela que los desastres naturales empeoran la desigualdad de ingresos en la región. Los resultados son significativos en las dos especificaciones del modelo, es decir, utilizando el coeficiente de Gini y el ingreso del primer quintil de la población para medir el nivel de desigualdades. Además, se observa que el efecto negativo de los desastres naturales sobre el nivel de desigualdad en la región se mantiene en el mediano plazo. Este resultado también es robusto a cambios en la metodología y en las variables utilizadas en el modelo. Luego, se emiten hipótesis sobre el impacto significativo de mediano plazo de los desastres naturales a partir de los trabajos existentes en la literatura reciente. Este trabajo constituye un aporte importante para el diseño de políticas públicas de reducción de la desigualdad en los países de la región.

Palabras clave: Desastres naturales, impacto económico, desigualdad de ingresos.

\section{Abstract}

This research paper explores the relationship between natural disasters and income inequality in Latin America and the Caribbean based on a panel data model covering 14 countries during the period 2000-2017. The econometric study revealed that natural disasters worsen income inequality in the region. The results are significant in the two specifications of the model that is, using the Gini coefficient or the income of the first quintile of the population to measure the level of inequalities. In addition, it is observed that the negative effect of natural disasters on the level of inequality is maintained in the medium term. hen, hypotheses are issued on the significant medium-term impact of natural disasters based on the existing works in the recent literature. This work constitutes an important contribution for the design of public policies to reduce inequality in the countries of the region. This result is also robust to changes in the methodology and in the variables used in the model.

Keywords: Natural disasters, economic impact, income inequality.

\section{Introducción}

Los desastres naturales tienen un impacto considerable en el desarrollo económico de los países. Inclusive, Barro (2006), demostró que la ocurrencia de desastres económicos imprevisibles como los conflictos armados o los desastres naturales de gran magnitud tiene mayores costos sociales que las fluctuaciones económicas continuas de menor amplitud. El impacto socioeconómico de los desastres naturales abarca diferentes dimensiones a nivel individual y agregado. Desde la perspectiva de un hogar, los desastres naturales pueden representar un choque adverso para el bienestar, afectando la integridad física, la acumulación de activos y los ingresos, mientras que desde una perspectiva agregada, pueden generar un impacto en el PIB debido a la pérdida de activos y la interrupción de la actividad económica. En las últimas décadas se ha observado un incremento de las investigaciones económicas sobre las consecuencias económicas de los desastres naturales, sin embargo, aún existen muchos vacíos en esta literatura (Cavallo y Noy, 2011, Kellenberg y Mobarak, 2011). Los proyectos de investigación recientes han evaluado el impacto de los desastres naturales en el crecimiento a corto y mediano plazo. Sin embargo, hay menos investigaciones sobre el impacto de los desastres naturales en los pobres y en la distribución del ingreso. En un estudio reciente, Yamamura (2013) utiliza datos de panel de 86 países durante el periodo 1965-2004 para analizar cómo los desastres naturales han afectado la desigualdad de ingresos. Concluye que los desastres naturales aumentan la desigualdad de ingresos en el corto plazo, sin embargo, este efecto decae con el tiempo y desaparece en el mediano plazo. Los resultados siguen estadísticamente significativos incluso después de controlar por los efectos fijos anuales y de países. Una de las hipótesis sugeridas por los autores para explicar la ausencia de efecto a mediano plazo es la redistribución de las riquezas de las áreas no dañadas a las áreas dañadas, lo que reduce la desigualdad de ingresos. Este resultado sigue la tendencia de la literatura empírica que distingue los efectos económicos de corto y de largo plazo de los desastres naturales. En efecto, si bien es cierto que la mayoría de los trabajos concluyen que los desastres naturales grandes tienen un impacto negativo a corto plazo en la dinámica de producción (Raddatz, 2009, y Noy, 2009), algunos estudios también encuentran una relación positiva entre desastres naturales y crecimiento a largo plazo (Skidmore y Toya, 2002), justificando estos resultados a partir de la teoría de la destrucción creativa. Otro canal de transmisión para explicar la desaparición del impacto de los desastres naturales consiste en el incremento del gasto público por parte de los gobiernos en la reconstrucción de las áreas afectadas por el desastre. Otro trabajo destacado es el de Islam y Winkel (2017), en el que se ofrece un marco analítico para analizar la relación entre cambio climático y desigualdad.

Evidencian que la relación se caracteriza por un círculo vicioso, en el cual la desigualdad inicial causa desventajas, es decir que los grupos más pobres sufrirán de manera desproporcionada los efectos adversos del cambio climático, lo que se traducirá en una mayor desigualdad posterior. Los autores identifican tres canales de transmisión a través de los cuales se materializa el efecto agravante sobre la desigualdad causada por el cambio climático: (i) el aumento de la exposición de los grupos desfavorecidos a los efectos adversos del cambio climático; (ii) el aumento de la susceptibilidad de los grupos desfavorecidos a los daños causados por el cambio climático; y (iii) la disminución de su capacidad para hacer frente y recuperarse del daño sufrido.

El estudio de la desigualdad ha ocupado un papel fundamental en las investigaciones sociales sobre los países de América Latina y el Caribe, desde que varias evidencias sugieren que América Latina y el Caribe aparece como la región con la distribución de los ingresos más desigual en el mundo (Alvaredo y Gasparini, 2015). En las últimas décadas, se observa un descenso estadísticamente significativo en los niveles de desigualdad del ingreso (CEPAL, 2017). Los principales factores que explican el descenso de la desigualdad son el aumento de los ingresos laborales de los trabajadores más pobres, es decir, una reducción en la desigualdad de ingresos laborales, el aumento en las transferencias gubernamentales y el cambio demográfico, que implica mayor número de miembros en edad de trabajar (Lustig et al, 2013, Azevedo et al., 2013). Sin embargo, a pesar de los avances de la región en la reducción de la pobreza y de la desigualdad en los últimos años, ambos problemas continúan siendo dos de los principales desafíos para los gobiernos de la región. De acuerdo con la información más reciente, la brecha entre las personas que se encuentran en los extremos de la distribución sigue a un nivel muy alto, pues el ingreso captado por el quintil más rico (quintil V) representa alrededor del $45 \%$ del ingreso de los hogares, mientras que el ingreso promedio del quintil de menores recursos (quintil I) es de apenas un 6\% de los ingresos totales (CEPAL, 2017).

Además, una de cada cuatro personas en la región sigue viviendo en la pobreza, y de los millones que han salido de la pobreza, la mayoría 
se encuentra en una situación de vulnerabilidad, es decir, que salieron de la pobreza moderada (ingresos inferiores a 4,00 USD al día), pero que continúan afrontando la inseguridad económica y, probablemente, nuevos periodos de pobreza en el futuro (Baez et al., 2017, Vakis et al, 2015). El coeficiente de Gini de la región es 4 puntos porcentuales más alto que el de África, 16 puntos más alto que el de Europa y Asia Central y 11 puntos más alto que el de China. Es más, 11 de los 20 países con más desigualdad de un total de 136, pertenecen a América Latina y el Caribe (Indicadores Mundiales de Desarrollo del Banco Mundial).

Los altos niveles de desigualdad y de pobreza se agravan por la vulnerabilidad de la región ante los riesgos de desastres naturales. En especial, en las últimas décadas, la región de América Latina y el Caribe sufrió varios desastres naturales de gran importancia, cuya magnitud, en términos de muertos y daños, ha provocado diferentes impactos en el ámbito económico y social, y ha acentuado el interés de los gobiernos nacionales para identificar de mejor manera las consecuencias de los desastres naturales para gestionar eficientemente los riesgos. El nivel de riesgo de desastres naturales para los países depende de tres dimensiones principales, la exposición, la vulnerabilidad (socioeconómica, grupos vulnerables) y la falta de capacidad (infraestructura e institucional). En el caso de la región, la exposición es fuerte, ya que las amenazas naturales como sequías, inundaciones, deslizamiento de tierras, tormentas, huracanes, terremotos y erupciones volcánicas constituyen un peligro constante para la región, pues la región se encuentra entre las regiones del mundo más propensas a las amenazas relacionadas con el clima, y se evidencia que el cambio climático aumentará la exposición de la región a los desastres relacionados con el clima (Mendelsohn and Williams, 2004). La vulnerabilidad de la región también es alta por los riesgos de origen humana en la región, resultado de una vulnerabilidad extrema, fruto de políticas inadecuadas de gestión de riesgos y del patrón de desarrollo socioeconómico de la región (Charvériat, 2000).

En las últimas décadas, el número de desastres naturales registrados ha aumentado significativamente, en especial los relacionados con el calentamiento global (Helmer et al., 2005). En América Latina y el Caribe, la ocurrencia de los desastres naturales sigue la misma tendencia creciente, como se observa en la tabla 1. En el periodo 1980-2019, ocurrieron en el mundo 12.561 desastres naturales, de los cuales un 23,9\% se produjeron en América (16,5\% en América Latina y el Caribe). Luego, los países latinoamericanos están en constante y siempre creciente peligro de amenazas naturales. Por una parte, la región se encuentra dentro de una de las regiones sísmicas más activas del mundo, que se traduce en terremotos frecuentes y severos. Algunos de los terremotos más violentos y destructivos registrados han ocurrido en la región (Haití, 2010, Chile, 2010, Ecuador, 2016, México, 2017). Por otra parte, América Central y el Caribe se encuentra en el camino de huracanes y tormentas tropicales. En promedio, al menos una isla caribeña es afectada por un ciclón tropical cada año (Baez et. al, 2017).

Tabla 1. Evolución de la cantidad de desastres naturales en América Latina y el Caribe

\begin{tabular}{lccccc}
\hline & $1970-1979$ & $1980-1989$ & $1990-1999$ & $2000-2009$ & $2010-2018$ \\
\hline Climatológico & 11 & 25 & 47 & 48 & 49 \\
Geofísico & 32 & 52 & 76 & 62 & 48 \\
Hidrológicos & 87 & 164 & 185 & 316 & 270 \\
Meteorológicos & 37 & 72 & 143 & 215 & 159 \\
Biológicos & 7 & 7 & 66 & 30 & 34 \\
\hline Total & 174 & 320 & 517 & 671 & 560 \\
\hline
\end{tabular}

Fuente: Base de Datos Internacional sobre Desastres (EM-DAT) del Centro de Investigación sobre la Epidemiología de Desastres (CRED)

La evolución de los desastres naturales, en incremento en la región, se acompaña de una tendencia creciente en las medidas de intensidad (figuras 2 y 3). Además, los indicadores de muertos y afectados por cada 1.000 habitantes están en aumento en la región, tanto para eventos de origen climático, geológico y tormentas (Bello, 2017). Por ese motivo, diferentes estudios en la región se han enfocado en analizar la relación entre pobreza y desastres naturales. En un estudio que incluye cinco países de América Latina y el Caribe, México, El Salvador, Perú, Bolivia y Ecuador, López y Ortíz (2009) sugieren que los desastres naturales suficientemente grandes o persistentes tienen un impacto nega- tivo tanto a corto plazo como a largo plazo e intergeneracional en la pobreza. De forma similar, Baez y Santos (2008) concluyen que en El Salvador los desastres naturales provocan un aumento en la profundidad y severidad de la pobreza. En el caso de Honduras, se evidenció que los activos de los hogares en el quintil de riqueza más bajo se redujeron en un $18 \%$, mientras que solamente se redujo en un $3 \%$ para el quintil de riqueza más alto después del huracán Mitch en el 1998 (Morris et al, 2002). En un trabajo que analiza el impacto de las sequías en el mercado laboral brasileño, Mueller y Osgood (2009) encontraron pérdidas salariales que van más allá del impacto inmediato de la sequía. Una reducción de una desviación estándar de precipitación puede reducir los salarios rurales de hasta un 18\% dentro de los 5 años y de un 9\% en los salarios dentro de los 5-10 años.

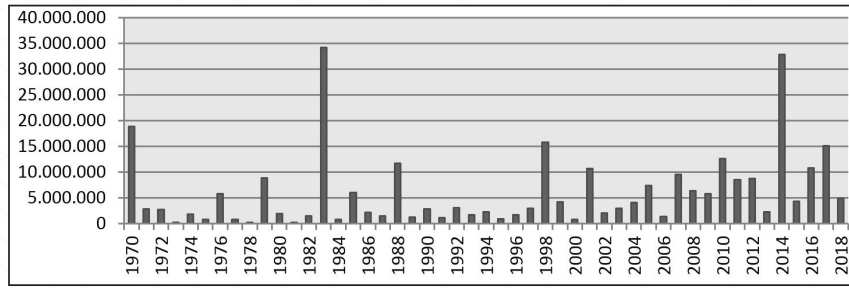

Figura 1. Total de afectados por desastres naturales en América Latina y el Caribe, durante el periodo 1970-2018

Fuente: Elaboración propia, a partir de la base de datos internacional sobre desastres(EM-DAT). El número total de afectados incluye heridos, afectados y sin hogares. Se calcula como la suma de los desastres climatológicos, geofísicos, hidrológicos y meteorológicos.

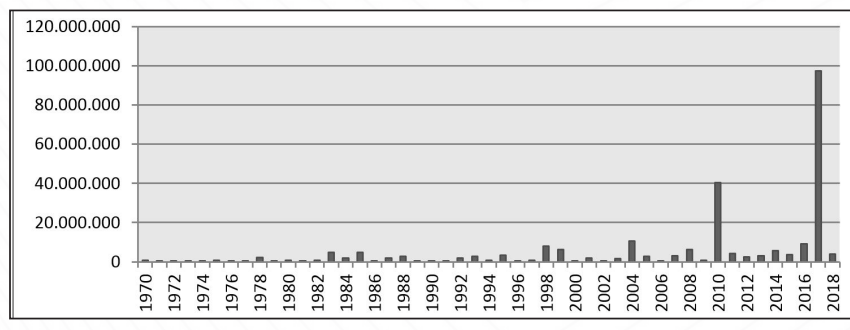

Figura 2. Daños económicos provocados por desastres naturales en América Latina ye Caribe durante el periodo 1970-2018

Fuente: Elaboración propia, a partir de la base de datos internacional sobre desastres (EM-DAT). El daño económico se calcula en 'OOO USD. Se calcula como la suma de los desastres climatológicos, geofísicos, hidrológicos y meteorológicos.

Tanto la pobreza como la desigualdad forman parte de los objetivos prioritarios en las políticas públicas, pues tiene efectos negativos en el crecimiento económico, la acumulación de los factores productivos y el capital humano. La vulnerabilidad de la región ante los riesgos de desastres naturales acentúa estos problemas. Tomando en cuenta estos acontecimientos, el objetivo de este trabajo es contribuir a estas discusiones al examinar la relación entre desastres naturales y desigualdad de ingresos en América Latina y el Caribe a partir de un modelo de datos de panel durante el periodo 2000-2017 y cuantificar el posible impacto de los desastres naturales, con el fin de contribuir a la elaboración de políticas púbicas de reducción de la desigualdad que toman en cuenta el riesgo climático. La principal pregunta de esta investigación es: ¿Cuál es el efecto a corto y mediano plazo de los desastres naturales sobre la desigualdad del ingreso en América Latina y el Caribe?

\section{Metodología}

La estimación econométrica se realizó utilizando datos de panel durante el periodo 2000-2017 para 14 países de América Latina y de Caribe. Chile, Guatemala y Nicaragua no están incluidos por falta de datos sobre la desigualdad de ingresos. Los datos son anuales. La metodología utilizada es similar a los trabajos en datos de panel que analizan los impactos de los desastres naturales sobre la pobreza (Yamamura, 2013). Dado que las variables de este estudio se miden de manera diferente, la desigualdad de ingresos se mide como un índice, el PIB per cápita en unidades monetarias, la apertura comercial, la inversión extranjera y el desarrollo del sector financiero en porcentaje del $\mathrm{PIB}$, y los desastres naturales en números de personas muertas, la transformación de las series de datos en logaritmos naturales ayuda a 
evitar los problemas asociados con sus problemas de distribución. La conversión de los datos en logaritmo es un enfoque más adoptado en la literatura empírica, ya que cada uno de los coeficientes estimados en el modelo de regresión puede interpretarse como elasticidades. Por ende, la ecuación para estimar es la siguiente:

$\ln \left(\right.$ Gini $\left._{i t}\right)=\beta_{0}+\beta_{1} \ln \left(\right.$ Desastres $\left._{i t}\right)+\beta_{2} \ln \left(\right.$ PIB $\left._{i t}\right)+\beta_{3} \ln \left(\right.$ Agricultura $\left._{i t}\right)+\beta_{4} \ln \left(\right.$ Apertura $\left._{i t}\right)$ $+\beta_{5} \ln \left(\right.$ Credito $\left._{i t}\right)+\beta_{6} \ln \left(F D I_{i t}\right)+\beta_{7} \ln \left(\right.$ Inflacion $\left._{i t}\right)+\beta_{8} \ln \left(\right.$ Corrupcion $\left._{i t}\right)+\beta_{9} \ln \left(\right.$ Tierra $\left._{i t}\right)$ $+\beta_{10} \ln \left(\right.$ Poblacion $\left._{i t}\right)+\eta_{i}+\varepsilon_{i t}$

En el modelo, la variable Gini , es una medida estándar de la desigualdad de ingreso, el coeficiente de Gini en el país i para el año t. Los datos sobre la desigualdad se han extraído de la base de datos de las Naciones Unidas sobre la desigualdad en el ingreso mundial (WIID4). Como variable alternativa para medir la desigualdad de ingresos se utilizó la participación en el ingreso del 20\% peor remunerado de la población (el quintil uno). Los datos provienen de los indicadores del Banco Mundial. La ocurrencia de desastres naturales proviene de la base de datos internacionales sobre desastres (EM-DAT), cuyos datos incluyen el número de afectados, número de muertos, y costo total de los desastres naturales. Para determinar el impacto en el corto y largo plazo de un desastre natural, el presente trabajo se enfoca no solamente en el cambio en el coeficiente de Gini en el periodo inmediatamente posterior a un desastre natural, sino en varios años después de los desastres naturales, por lo tanto, se incluyen variables retrasadas de los desastres naturales. Se utiliza el número de muertos para medir el impacto de un desastre natural. Además, para tomar en cuenta el nivel de

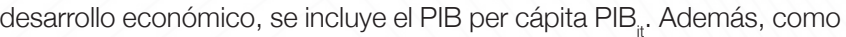
se prevé que los países de mayor tamaño experimentarán más desastres naturales si todos los demás parámetros se mantienen iguales, se incluye el tamaño del país como variable explicativa (Tierra ${ }_{i t}$ ), sino, el número de desastres naturales se correlacionará con el término de error si no se controla por el tamaño. Conforme a lo que establecen otros estudios, el modelo también incluye las siguientes variables: la participación de la agricultura en la producción total (agricultura $)$, la apertura de la economía (apertura), la inversión extranjera directa, entradas netas, en porcentaje del PIB (FDI $)$, la inflación anual (Inflación. $)$, y el desarrollo financiero, medido como los créditos otorgados al sector privado por los bancos comerciales y otras instituciones financieras, en porcentaje del PIB (Credito ${ }_{\mathrm{it}}$ ). Estas variables provienen de los Indicadores de Desarrollo Mundial del Banco Mundial. Además, el modelo incluye el nivel de corrupción (Corrupción ${ }_{\text {it }}$ ), proveniente de los indicadores de gobernanza mundial del Banco Mundial. Además, en la ecuación (1), $\eta$ es el efecto fijo especifico del país, ßi son los coeficientes que deben estimarse, $e_{i t}$ es el término de error estocástico, i y t indexan respectivamente los países y los años. La tabla 1 resume las principales variables y sus fuentes de información.

\begin{tabular}{|c|c|}
\hline Variable & Fuente de información \\
\hline Coeficiente de Gini & $\begin{array}{l}\text { Base de datos de las Naciones Unidas sobre la } \\
\text { desigualdad en el ingreso mundial (WIID4) }\end{array}$ \\
\hline $\begin{array}{l}\text { Ingreso del } 20 \% \text { peor remunerado de la } \\
\text { población }\end{array}$ & Indicadores del Desarrollo Mundial - Banco Mundial \\
\hline Número de muertos por desastres naturales & $\begin{array}{l}\text { Base de datos internacional sobre desastres (EM- } \\
\text { DAT) }\end{array}$ \\
\hline $\begin{array}{l}\text { PIB per Cápita } \\
\text { Participación de la agricultura en la } \\
\text { producción total }\end{array}$ & $\begin{array}{l}\text { Indicadores del Desarrollo Mundial - Banco Mundial } \\
\text { Indicadores del Desarrollo Mundial - Banco Mundial }\end{array}$ \\
\hline $\begin{array}{l}\text { Inversión extranjera directa, entradas netas, } \\
\text { en porcentaje del PIB }\end{array}$ & Indicadores del Desarrollo Mundial - Banco Mundial \\
\hline Apertura de la economía & Indic \\
\hline $\begin{array}{l}\text { Población } \\
\text { Créditos otorgados al sector privado por los }\end{array}$ & ndial \\
\hline $\begin{array}{l}\text { bancos comerciales y otras instituciones } \\
\text { financieras, en porcentaje del PI }\end{array}$ & Indicadores del Desarrollo Mundial - Banco Mundial \\
\hline Inflación anual & rrollo Mundial - \\
\hline Nivel de corrupción & Indicadores del Desarrollo Mundial - Banco Mundial \\
\hline
\end{tabular}

Fuente: Elaboración propia

\section{Resultados}

Los resultados de la ecuación (1) se presentan en la tabla 2. Se estiman diferentes especificaciones en las columnas (1) a (4). La prueba de Hausman rechaza el modelo de efectos aleatorios en favor del modelo de efectos fijos. La columna (1) indica los resultados de la estimación principal. En la columna (2), se agregan variables retrasadas de los desastres naturales con el fin de determinar el impacto de mediano plazo, en la columna 3 variables retrasadas de la variable dependiente, y en la columna 4 variables retrasadas tanto de la variable dependiente como de los desastres naturales. Los resultados empíricos evidencian que

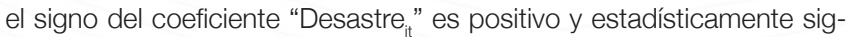
nificativo en la columna (1). Eso es, los desastres naturales provocan un aumento de la desigualdad de ingresos, medido por el coeficiente de Gini. También se encuentra un coeficiente positivo y significativo en las especificaciones de los modelos alternativos en las otras columnas de la tabla 2, lo que confirma que el impacto negativo de los desastres naturales sobre el nivel de desigualdad en el país es significativo. Luego, las columnas (2) y (4) permiten concluir sobre el impacto de mediano plazo, mediante la incorporación de variables retrasadas de los desastres naturales. De nuevo, el impacto negativo sigue estadísticamente significativo, aunque a un nivel de significatividad inferior, al nivel de $10 \%$ en vez de 5\%. Por ende, los resultados subrayan que los desastres naturales en América Latina y el Caribe aumentan la desigualdad de ingresos y este resultado persiste en el mediano plazo.

El PIB per capita tiene el signo esperado y es estadísticamente significativo. Los resultados demuestran que el crecimiento económico en la región en las últimas décadas ha sido inclusivo. Además, eso rechaza la hipótesis de Kuznets que establece que a medida que aumente el desarrollo de un país, el ingreso se concentra y la distribución del ingreso empeora. Diferentes estudios han demostrado que el progreso social en América Latina y el Caribe en las últimas décadas ha permitido el incremento de la inversión pública en infraestructura, servicios básicos y programas sociales, contribuyendo así a los objetivos de reducción de pobreza (Székely, 2015). La participación de la agricultura en la producción total tiene un signo negativo y es estadísticamente significativo, lo que significa que a medida que aumenta la participación de la agricultura en la economía, la desigualdad disminuye. En cuanto a las otras variables del modelo, se observa que el coeficiente relacionado con el desarrollo financiero es estadísticamente significativo y positivo, lo que significa que el desarrollo del sector financiero se asocia con un incremento de la desigualdad. Resultados similares fueron encontrados por Morley (2000) y Berry (1998). Las otras variables tienen los signos esperados pero no son estadísticamente significativas. La apertura comercial, la corrupción y la inversión extranjera tienden a aumentar la desigualdad de ingresos en América Latina y el Caribe.

La tabla 3 presenta los resultados de la ecuación (1) cambiando únicamente la variable dependiente. Se utiliza como medida alternativa la participación en el ingreso del $20 \%$ peor remunerado de la población (el quintil uno). El signo del coeficiente "Desastre ," es negativo y estadísticamente significativo en la columna (1), lo que significa que los desastres naturales disminuyen la participación en el ingreso del primer quintil. En otras palabras, se confirma que los desastres naturales empeoran la desigualdad. En cuanto al impacto de mediano plazo, los resultados de las columnas (2) y (4) evidencian que el impacto negativo de los desastres naturales sigue estadísticamente significativo a un nivel de $5 \%$. Por ende, los resultados de la tabla 3 se interpretan como un test de robustez que respalda los hallazgos de la tabla 2.

En cuanto a las otras variables económicas del modelo, los resultados coinciden con los resultados anteriores. El crecimiento del producto interno bruto per cápita incrementa la participación en el ingreso del primer quintil, confirmando el crecimiento inclusivo que ha experimentado la región en el siglo XXI. La participación de la agricultura en el PIB tiene un signo positivo y es estadísticamente positivo, y el desarrollo del sistema financiero contribuye a incrementar las desigualdades. A diferencia de la tabla anterior, la corrupción es estadísticamente significativa y con un coeficiente negativo, es decir, la corrupción incrementa la desigualdad. Los coeficientes de las otras variables son consistentes con los resultados de la tabla 2 pero no llegan a ser significativos. 
Tabla 3. Resultados de las estimaciones utilizando el coeficiente de Gini como variable dependiente

\begin{tabular}{|c|c|c|c|c|}
\hline Variables & (1) & (2) & (3) & (4) \\
\hline \multirow{2}{*}{$\ln ($ Ginit-1) } & & & $0,007^{* * *}$ & $0,007^{* * *}$ \\
\hline & & & 4,22 & 3,80 \\
\hline \multirow{2}{*}{ Desastrest } & $0,025^{*}$ & $0,024^{*}$ & $0,033^{* *}$ & $0,021^{*}$ \\
\hline & 1,84 & 1,81 & 2,55 & 1,71 \\
\hline \multirow{2}{*}{ Desastrest-1 } & & 0,059 & & $-0,003$ \\
\hline & & 0,40 & & $-0,22$ \\
\hline \multirow{2}{*}{ Desastrest-2 } & & $0,026^{*}$ & & $0,0244^{*}$ \\
\hline & & 1,72 & & 1,83 \\
\hline \multirow{2}{*}{ Desastrest-3 } & & $0,031^{* *}$ & & 0,017 \\
\hline & & $\begin{array}{c}2,07 \\
0,026^{*}\end{array}$ & & $\begin{array}{c}1,29 \\
0,023^{*}\end{array}$ \\
\hline Desastrest -4 & & 1,94 & & 1,79 \\
\hline \multirow{2}{*}{$\ln \left(\mathrm{PlBit}_{\mathrm{it}}\right)$} & $-0,998^{* * *}$ & $-0,068^{* * *}$ & $-0,759^{* * *}$ & $-0,057^{\text {*** }}$ \\
\hline & $-6,35$ & $-0,86$ & $-5,03$ & $-3,19$ \\
\hline \multirow{2}{*}{$\ln \left(\mathrm{Agr}_{\mathrm{it}}\right)$} & $-0,054^{* *}$ & $-0,021$ & $-0,059^{* * *}$ & $-0,026$ \\
\hline & $-2,44$ & 0,96 & $-3,02$ & $-1,14$ \\
\hline \multirow{2}{*}{$\ln \left(\right.$ Aper $\left._{\mathrm{it}}\right)$} & 0,003 & 0,024 & 0,004 & 0,009 \\
\hline & 0,14 & 0,81 & 0,18 & 0,42 \\
\hline \multirow[b]{2}{*}{$\ln \left(\right.$ Cred $\left._{t t}\right)$} & $0,044^{* *}$ & 0,017 & $0,032^{* *}$ & 0,015 \\
\hline & 2,37 & 0,20 & 2,01 & 0,82 \\
\hline \multirow{2}{*}{$\ln \left(\mathrm{FDI}_{\mathrm{it}}\right)$} & 0,020 & 0,003 & 0,019 & 0,003 \\
\hline & 1,62 & 0,2 & 1,57 & 0,24 \\
\hline \multirow{2}{*}{$\ln$ (Inflacion) } & 0,000 & $-0,007$ & $-0,010$ & $-0,006$ \\
\hline & 0,2 & $-0,75$ & $-1,37$ & $-0,69$ \\
\hline \multirow{2}{*}{$\ln ($ Corrit) } & 0,001 & 0,014 & 0,005 & 0,014 \\
\hline & 0,13 & 1,27 & 0,78 & 1,55 \\
\hline \multirow{2}{*}{$\ln$ (Paísit) } & $0,703^{* * *}$ & $0,880^{* * *}$ & $0,582^{* * *}$ & $0,602^{* * *}$ \\
\hline & 6,87 & 5,94 & 5,80 & 4,20 \\
\hline \multirow{2}{*}{$\ln$ (Poblit) } & $-0,329^{* * *}$ & $-0,497^{* * *}$ & $-0,254^{* * *}$ & $-0,282^{* * *}$ \\
\hline & $-3,55$ & $-3,80$ & $-2,97$ & $-2,33$ \\
\hline $\mathrm{R}^{2}$ & 0,99 & 0,99 & 0,96 & 0,97 \\
\hline Observaciones & 226 & 177 & 212 & 176 \\
\hline
\end{tabular}

Fuente: Elaboración propia, la variable dependiente es el coeficiente de Gini.. Todas las variables se encuentran en su forma logarítmica (excepto las variables "dummy"). El signo de notificación de *, ${ }^{* *} y^{* * *}$ denota el nivel significativo de 10\%, 5\% y $1 \%$ respectivamente. El valor dentro del paréntesis () se refiere al estadístico t para el modelo de efectos fijos.

Tabla 4. Resultados de las estimaciones utilizando el ingreso del primer quintil como variable dependiente

\begin{tabular}{|c|c|c|c|c|}
\hline Variables & (1) & (2) & (3) & (4) \\
\hline \multirow{2}{*}{$\ln \left(\right.$ Quintill $\left.1_{t-1}\right)$} & & & 0,019 & $0,335^{* * * *}$ \\
\hline & & & 0,12 & 3,38 \\
\hline \multirow[t]{2}{*}{ Desastrest } & $-0,100 * *$ & $-0,070^{* *}$ & $-0,069^{*}$ & $-0,126^{* * *}$ \\
\hline & $-2,14$ & $-2,05$ & $-1,77$ & \\
\hline \multirow{3}{*}{ Desastrest-1 } & & $-0,017$ & $-0,015$ & \\
\hline & & $-0,46$ & $-0,36$ & \\
\hline & & $-0,093^{* *}$ & $-0,095^{* *}$ & \\
\hline Desastrest-2 $_{2}$ & & $-2,34$ & $-2,22$ & \\
\hline \multirow{2}{*}{ Desastrest-3 } & & $-0,121 * * *$ & $-0,130^{* *}$ & \\
\hline & & $\begin{array}{c}-3,21 \\
-0,097 * *\end{array}$ & $\begin{array}{c}-2,52 \\
-0,102^{* *}\end{array}$ & \\
\hline Desastrest-4 & & $-2,52$ & $-2,48$ & \\
\hline \multirow{2}{*}{$\ln \left(\mathrm{PIB}_{\mathrm{it}}\right)$} & $0,22^{* * *}$ & $0,129 * * *$ & $0,133^{* *}$ & $0,161^{* * *}$ \\
\hline & 4,68 & 2,88 & 2,30 & 3,89 \\
\hline \multirow{2}{*}{$\ln (\mathrm{Agrit})$} & $0,205^{* * *}$ & $0,159 * *$ & $0,184 * *$ & $0,223^{* * *}$ \\
\hline & 3,06 & 2,53 & 2,59 & 4,27 \\
\hline \multirow{2}{*}{$\ln ($ Aperit $)$} & $-0,007$ & $-0,063$ & $-0,053$ & $-0,033$ \\
\hline & $-0,08$ & $-0,85$ & $-0,76$ & $-0,60$ \\
\hline \multirow{2}{*}{$\ln \left(\right.$ Cred $\left._{i t}\right)$} & $-0,209 * * *$ & $-0,010$ & $-0,015$ & $-0,114^{* * *}$ \\
\hline & $-4,08$ & $-0,15$ & $-0,27$ & $-3,12$ \\
\hline \multirow{2}{*}{$\ln \left(\mathrm{FDI}_{\mathrm{it}}\right)$} & $-0,063$ & 0,002 & 0,001 & $-0,036$ \\
\hline & $-1,41$ & 0,07 & 0,04 & $-0,93$ \\
\hline \multirow{2}{*}{$\ln$ (Inflacion) } & $-0,007 * *$ & 0,030 & 0,036 & 0,037 \\
\hline & $-2,17$ & 0,94 & 1,15 & 1,48 \\
\hline \multirow{2}{*}{$\ln \left(\right.$ Corr $\left._{\mathrm{it}}\right)$} & $-0,022$ & $-0,074^{* *}$ & $-0,081^{* * *}$ & $-0,032^{* *}$ \\
\hline & $-1,06$ & $-2,51$ & $-2,64$ & $-2,06$ \\
\hline \multirow{2}{*}{$\ln$ (Paísit) } & $-1,850^{* * *}$ & $-1,730^{* * *}$ & $-1,818^{* * *}$ & $-1,312^{* * *}$ \\
\hline & $-5,44$ & $-3,88$ & $-3,76$ & $-4,17$ \\
\hline \multirow{2}{*}{$\ln \left(\right.$ Poblit $\left._{i}\right)$} & $1,548^{* * *}$ & $1,470^{* * *}$ & 1,536 *** & $1,077^{* * *}$ \\
\hline & 5,03 & 3,66 & 3,69 & 3,92 \\
\hline $\mathrm{R}^{2}$ & 0,84 & 0,77 & 0,82 & 0,88 \\
\hline Observaciones & 161 & 207 & 155 & 187 \\
\hline
\end{tabular}

Fuente: Elaboración propia, la variable dependiente es el coeficiente de Gini. . Todas las variables se encuentran en su forma logarítmica (excepto las variables "dummy"). El signo de notificación de *, ${ }^{* *} y^{* * *}$ denota el nivel significativo de $10 \%, 5 \%$ y $1 \%$ respectivamente. El valor dentro del paréntesis () se refiere al estadístico t para el modelo de efectos fijos.

\section{Conclusiones}

A partir de un modelo de panel cubriendo 14 países de la región latinoamericana durante un periodo de 17 años (2000-2017), los resultados concluyen que los desastres naturales empeoran el nivel de desigualdad de ingresos en América Latina y el Caribe. Los resultados son significativos en las dos especificaciones del modelo, es decir, utilizando el coeficiente de Gini o el ingreso del primer quintil de la población para medir el nivel de desigualdades. Las otras variables también coinciden con los resultados empíricos previos, principalmente, se encuentra que el crecimiento económico en la última década permitió reducir las desigualdades en la región.
Además, se observa que el efecto negativo de los desastres naturales sobre el nivel de desigualdad se mantiene en el mediano plazo. Este resultado también es robusto a cambios en la metodología y en las variables utilizadas en el modelo. Cuando se agregan variables retrasadas de los desastres naturales, el coeficiente negativo se mantiene estadísticamente significativo durante cuatro años. Esto contribuye a alimentar el debate sobre el impacto de largo plazo de los desastres naturales. El impacto de mediano plazo de los desastres naturales sobre la desigualdad de ingresos es un resultado nuevo en la literatura de los desastres naturales.

Los resultados discutidos en la sección anterior permiten emitir hipótesis sobre el impacto significativo de mediano plazo. La hipótesis principal que explica el impacto de mediano plazo es que, como se evidenció en la revisión de la literatura empírica, en América Latina y el Caribe los hogares más pobres tienen más probabilidad de registrar una tendencia persistente de bajo crecimiento de los ingresos, mientras que los hogares con mayores ingresos tienden a recuperarse más fácilmente. En muchas ocasiones los ingresos de los hogares expresan grandes caídas después de los fenómenos naturales, en especial en los sectores rurales que dependen principalmente de la agricultura (Morris et. al, 2001, Ishizawa y Miranda, 2016, Caruso, 2015). Además, no todos los hogares se recuperan de la misma manera después de una catástrofe natural, en especial, en el caso de los hogares pobres y vulnerables, la recuperación económica puede ser más larga. Luego, existen varias evidencias que demuestran que los hogares más pobres tienen más probabilidad de registrar una tendencia persistente de bajo crecimiento de los ingresos, mientras que los hogares con mayores ingresos tienden a recuperarse más fácilmente. Es decir, la incapacidad de los hogares pobres de poder mitigar los efectos adversos de los fenómenos naturales puede empujar al alza la desigualdad de ingresos en el corto y largo plazo. Otra de las interpretaciones posibles radica en el comportamiento procíclico de las políticas fiscales en América Latina, lo que puede impedir los esfuerzos en tema de gasto público de los gobiernos después de los desastres naturales. Además, el impacto de los desastres naturales varía según sus características. Por ejemplo, en algunas zonas, se producen tormentas varias veces al año y suelen seguir el mismo patrón, por lo que la gente puede estar preparada.

En cambio, un terremoto o inundación, menos frecuentes y previsibles, pueden provocar un costo económico más alto. Por ende, se recomienda analizar a futuro el impacto diferenciado de los desastres naturales.

El estudio constituye un resultado importante para los gobiernos en e diseño de políticas públicas. En efecto, los desastres naturales afectan de manera negativa los logros que ha conseguido la región en cuanto a la reducción de la pobreza y de la desigualdad de ingresos y se requiere definir políticas públicas que permitan reducir la exposición y vulnerabilidad de las poblaciones más pobres a los desastres naturales. Las políticas para la prevención de los desastres naturales deben ser diseñadas para reducir los factores que determinan la vulnerabilidad de las comunidades, el grado de exposición, la protección, la reacción inmediata, la recuperación básica y la reconstrucción, tomando en cuenta el riesgo específico local y la incidencia de las problemáticas sociales como la pobreza y el nivel de desigualdad.

\section{Referencias}

Alvaredo, F. \& Gasparini, L. (2015). Recent trends in inequality and poverty in developing countries", Handbook of Income Distribution. A. Atkinson y F. Bourquignon (eds.), vol. 2, Amsterdam, Elsevier.

Azevedo, J. P., Inchauste, G., Olivieri, S., Saavedra, J., \& Winkler, H. (2013). Is labor income responsible for poverty reduction? A decomposition approach. The World Bank.

Baez, J. E., \& Santos, I. V. (2008). On shaky ground: The effects of earthquakes on household income and poverty. RPP LAC-MDGs and Poverty-02/2008, RBLAC-UNDP, New York.

Baez, J. E., Rodriguez-Castelan, C., \& Fuchs, A. (2017). ¿Desarrollo Económico Inestable? Choques Agregados en América Latina y el Caribe. The World Bank. 
Barro, R. J. (2006). Rare disasters and asset markets in the twentieth century. The Quarterly Journal of Economics, 121(3), 823-866.

Bello, O. (2017). Desastres, crecimiento económico y respuesta fiscal en los países de América Latina y el Caribe, 1972-2010. Revista Cepal.

Berry, A. (1998). Poverty, economic reform \& income distribution in Latin America. Boulder, Colorado, Lynne Rienner.

Caruso, G., \& Miller, S. (2015). Long run effects and intergenerational transmission of natural disasters: A case study on the 1970 Ancash Earthquake. Journal of development economics, 117, 134-150.

Cavallo, E., \& Noy, I. (2011). Natural disasters and the economy - a survey. International Review of Environmental and Resource Economics, 5(1), 63102.

Charveriat, C. (2000). Natural disasters in Latin America and the Caribbean: An overview of risk. Working paper 434, Inter-American Development Bank, Washington DC.

Comisión Económica para América Latina y el Caribe (CEPAL). Panorama Social de América Latina, 2017 (LC/PUB.2018/1-P). Santiago, 2018

Helmer, M \& Hilhorst, D. (2006). Natural disasters and climate change. Disasters, 30(1), 1-4.

Ishizawa, O. A., \& Miranda, J. J. (2016). Weathering storms: understanding the impact of natural disasters on the poor in Central America. The World Bank.

Islam, S. N., \& Winkel, J. (2017). Climate change and social inequality. United Nations, Department of Economic and Social Affairs.

Kellenberg, D. K., \& Mobarak, A. M. (2008). Does rising income increase or decrease damage risk from natural disasters?. Journal of urban economics, 63(3), 788-802.

López-Calva, LF and E Ortiz-Juárez (2009). Evidence and Policy Lessons on the Links between Disaster Risk and Poverty in Latin America: Methodology and Summary of Country Studies. Research for Public Policy, MDGs and Poverty, MDG-01-2009, RBLAC-UNDP, New York.

Lustig, N., Lopez-Calva, L. F., Ortiz-Juarez, E., \& Monga, C. (2016). Deconstructing the decline in inequality in Latin America. In Inequality and growth: Patterns and policy (pp. 212-247). Palgrave Macmillan, London.

Mendelsohn, R., \& Williams, L. (2004). Comparing forecasts of the global impacts of climate change. Mitigation and Adaptation Strategies for Global Change, 9(4), 315-333.

Morley, S. A. (2000). The effects of growth and economic reform on income distribution in Latin America. Cepal Review.

Morris, S. S., Neidecker-Gonzales, O., Carletto, C., Munguía, M., Medina, J. M., \& Wodon, Q. (2002). Hurricane Mitch and the livelihoods of the rural poor in Honduras. World development, 30(1), 49-60.

Mueller, V. A., \& Osgood, D. E. (2009). Long-term impacts of droughts on labour markets in developing countries: evidence from Brazil. The Journal of Development Studies, 45(10), 1651-1662.

Noy, I. (2009). The macroeconomic consequences of disasters. Journal of Development economics, 88(2), 221-231.

Raddatz, C. (2009). The wrath of God: macroeconomic costs of natural disasters. The World Bank.

Skidmore, M., \& Toya, H. (2002). Do natural disasters promote long-run growth?. Economic inquiry, 40(4), 664-687.

Székely, M., \& Mendoza, P. (2015). Is the decline in inequality in Latin America here to stay?. Journal of Human Development and Capabilities, 16(3), 397-419.

Vakis, R. N. (2006). Complementing natural disasters management: the role of social protection. World Bank, Social Protection.

Yamamura, E. (2013). Impact of natural disasters on income inequality: Analysis using panel data during the period 1965 to 2004. Munich Personal RePEc Archive. 